\title{
Laser Short-Pulse Heating: Influence of Spatial Distribution of Absorption Coefficient on Temperature Field in Silicon Film
}

\author{
S. B. Mansoor ${ }^{*}$ and B. S. Yilbas ${ }^{* *}$ \\ *Mechanical Engineering Department, King Fahd University of Petroleum \& Minerals, Mail Box 1302, Dhahran 31261, \\ Saudi Arabia \\ E-mail: saadbin@kfupm.edu.sa \\ ${ }^{* *}$ Mechanical Engineering Department, King Fahd University of Petroleum \& Minerals, Mail Box 1913, Dhahran 31261, \\ Saudi Arabia \\ E-mail:bsyilbas@kfupm.edu.sa
}

Laser short-pulse heating of silicon film is considered and effect of the spatial distribution of absorption coefficient on electron and lattice site temperatures is examined. The high absorption region in the silicon film resembles the presence of absorbed particles in this region, since the absorption coefficient of silicon at the wavelength of the laser irradiation is significantly low. Electron and lattice temperatures are predicted using the electron kinetic theory approach. Three different spatial distributions of absorption coefficient are considered in the simulations. It is found that electron temperature attains the highest for the case of high absorption coefficient located in the surface region of the silicon film. As the high absorption region moves inside the film, electron and lattice temperatures become low.

DOI:10.2961/jlmn.2012.02.0009

Keywords: laser, short-pulse, absorption, silicon film.

\section{Introduction}

High intensity short-pulse laser heating results in non-equilibrium energy transport in the solid substrates. However, some of the solids have low absorption coefficient for the incident radiation. This results in low energy deposition and small temperature rise in the electron and lattice sub-systems. The partially absorbing films can be engineered to improve the absorption coefficient using the metallic nano particles in the structure. In this case, the distribution of nano particles can be arranged to obtain the desired absorption within the film. However, the deposition of laser energy through absorption in the nano structured film enhances temperature rise and improves the laser machining. Consequently, investigation into the energy transfer in the electron and lattice sus-bsystems of partially absorbing films with the presence of nano particles becomes necessary.

Considerable research studies were carried out to examine the non-equilibrium heating process. The nonequilibrium electron heating in copper was examined by Eesley [1]. He proposed and used a thermomodulation spectroscopy, which permitted the measurement of the separation of electronic and lattice contributions to the heating-induced-reflectivity change. Brorson et al. [2] studied the electronic heat-transport dynamics in thin gold films during femtosecond irradiation. They suggested that the ballistic electronic motion could contribute to heat transport in short time and the small length scales considered. Wang et al. [3] carried out electron temperature measurement in a highly excited gold substrate incorporating the femtosecond thermionic emission. They showed that thermophysical properties, such as thermal conductivity, electron-phonon coupling, and electronic specific heat capacity, at high temperature were significantly different than those corresponding to the room temperature values. The partial coherence of thin film radiative properties was examined by Chen et al. [4]. They presented the expressions for the normal reflectance and transmittance of a thin film not only degenerate into the limiting results of the wave and geometric optics in the coherent and incoherent cases, but applied for all partial coherent states between the limits. Malinowski [5] examined the relaxation model for heat conduction and generation due to the finite velocity of heat propagation and the inertia of the internal heat source. He indicated that the relaxation solutions, in general, did not tend to approach the corresponding parabolic solutions. Laser heating of multi-layer metals was examined by Qiu et al. [6]. They indicated that the conventional radiation heating model fails to predict the correct energy transfer in the electron and lattice subsystems, since radiation absorption by free electrons and the subsequent heating of the lattice occurred at different locations in a multi-layer metal film. The ultrashort laser pulse absorption in solid targets was investigated by Pozmers et al. [7]. They presented the absorption coefficient from the plasma conductivity, which included the physical processes responsible for collisional and collisionless skin layer absorption mechanisms. The non-equilibrium heating of metal films was examined by Al-Nimer and Mosoud [8]. They showed that the perturbation technique could be used to eliminate the coupling between the two energy equations when the temperature difference between the electron gas and lattice became small. The wave diffusion and parallel nonequilibrium heat conduction in solids were examined by Honner and Kunnes [9]. They introduced dimensionless criteria for micro-scale heat transfer after incorporating the criteria used for the equilibrium diffusion conduction. The improved two-temperature model and its application in ultra-short laser heating of metal films was introduced by Jiang and Tsai [10]. They incorporated the quantum approach to calculate the substrate properties including the 
electron heat capacity, electron relaxation time, electron conductivity, and absorption coefficient. A new numerical scheme for simulating multidimensional transient and steady-state micro-scale energy transport was developed by Pilon and Katika [11]. They showed that predictions agreed well with the analytical solutions. The finite element simulations for short pulse laser radiative transfer in homogenous and nonhomogenous media were carried out by An et al. [12]. They indicated that the reflected and transmitted signals could not be solely taken as the experimental data to predict the optical properties of the medium.

In the present study, laser short-pulse heating of silicon film with the variable absorption coefficient due to the presence of nano particles at different locations in the film is examined. The spatial variation of the absorption coefficient is considered to resemble the particle distributions at different locations in the film. Aluminium plate is considered adjacent to the silicon film. Electron and lattice temperatures are predicted using the electron kinetic theory for three spatially varying absorption coefficients within the silicon film.

\section{Heating Analysis}

Laser short-pulse heating of silicon layer situated at the top of gold substrate is considered. Figure (1) shows the silicon film and the gold layer. Energy transfer in lattice and electron sub-systems can be formulated after considering the electron kinetic theory approach [13 - 15]. Consequently, the resulting equations are [13 - 15]:

$$
\begin{aligned}
& A \frac{\partial T_{e}}{\partial t}=B \nabla^{2} T_{e}-C\left[T_{e}-T_{l}\right]+I_{0} \delta \exp (-\delta|x|) \\
& D \frac{\partial T_{l}}{\partial t}=C\left[T_{e}-T_{l}\right]
\end{aligned}
$$

where : A, B, C, and D are the coefficients, which are [1315]:

$$
\begin{aligned}
& A=\frac{f k \tau_{s}}{\lambda^{2}}=C_{e}: B=k: C=\frac{f k}{\lambda^{2}}\left(1-\frac{f k \tau_{s}}{\rho C_{p} \lambda^{2}}\right)=G \\
& D=C_{l}-\frac{f k \tau_{s}}{\lambda^{2}}=C_{l}-C_{e}
\end{aligned}
$$

\subsection{The laser Source Term}

The laser source term in eq. 1 is selected to resemble the laser output pulse, which was used in the experiment [19], i.e. the laser was colliding-pulse modelocked (CPM) dye laser with a wavelength $630 \mathrm{~nm}$. The laser source term is, therefore:

$$
I(t)=0.94 \frac{1-r_{f}}{t_{p}} \alpha J \exp \left(-x \alpha-2.77\left(\frac{t}{t_{p}}\right)^{2}\right)
$$

where $t_{p}$ is full width-at-half-maximum (FWHM) duration of the laser pulse, and $t=0$ is defined at the moment when the peak of a laser pulse arrives at the metal surface. $\mathrm{J}$ is the total energy carried by a laser pulse divided by the laser spot cross section. In the simulations, $t_{p}=96$ femtosecond, $\mathrm{J}=150 \mathrm{~J} / \mathrm{m}^{2}$, and $\mathrm{r}_{\mathrm{f}}: 0.94$ are employed. Figure (2) shows the temporal distribution of the laser pulse. Moreover, the peak laser intensity is determined from $I_{o}=\frac{0.94 \times J}{t_{p}}$. The time span of the laser pulse distribution starts from zero to its peak value. However, in the present formulation, the time span starts from the peak intensity; in which case at $t=0$ then $I(0)$ becomes the maximum.

\subsection{Absorption of Laser Intensity}

The laser intensity in a one-dimensional thin film, at any point in it is given by the Beer-Lambert's Law:

$\frac{d I}{d x}=-\alpha I$

where $x$ is the depth along the thin film in units of $m$, $I=I(x)$ is the laser intensity at a depth $x$ in units of $W / \mathrm{m}^{2}$, and $\alpha$ is the absorptivity or the absorption coefficient of the material in units of $\mathrm{m}^{-1}$. The surface of the thin film lies at $x=0$. The absorption coefficient can be variable within the substrate material; in which case, the laser intensity can be a function of $x$. It can be obtained after integrating eq. 3 ,.which is:

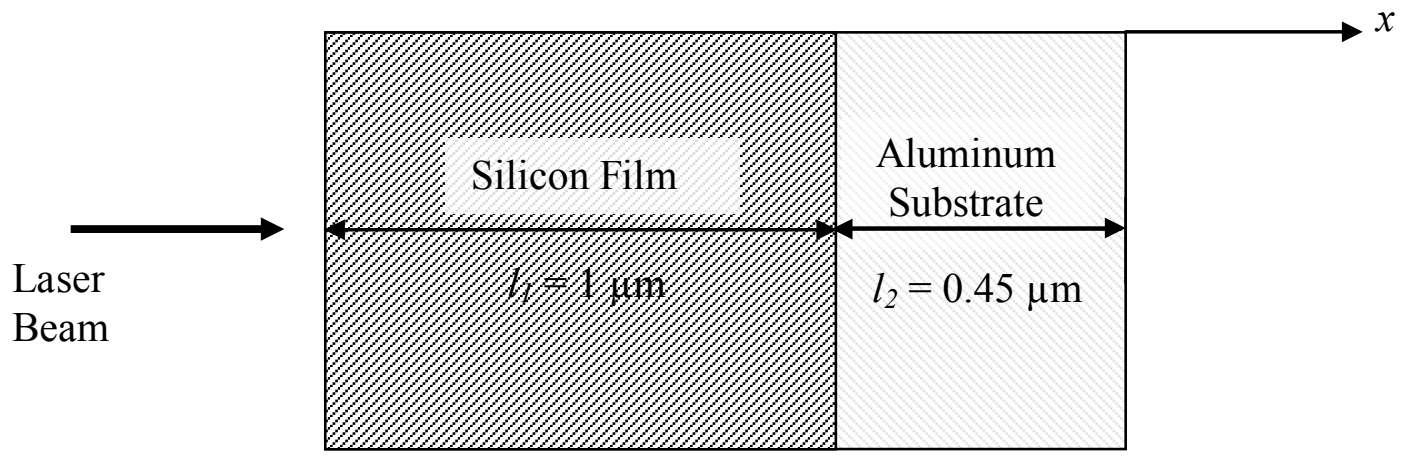

Fig. 1 A schematic view of silicon layer and aluminum substrate. 


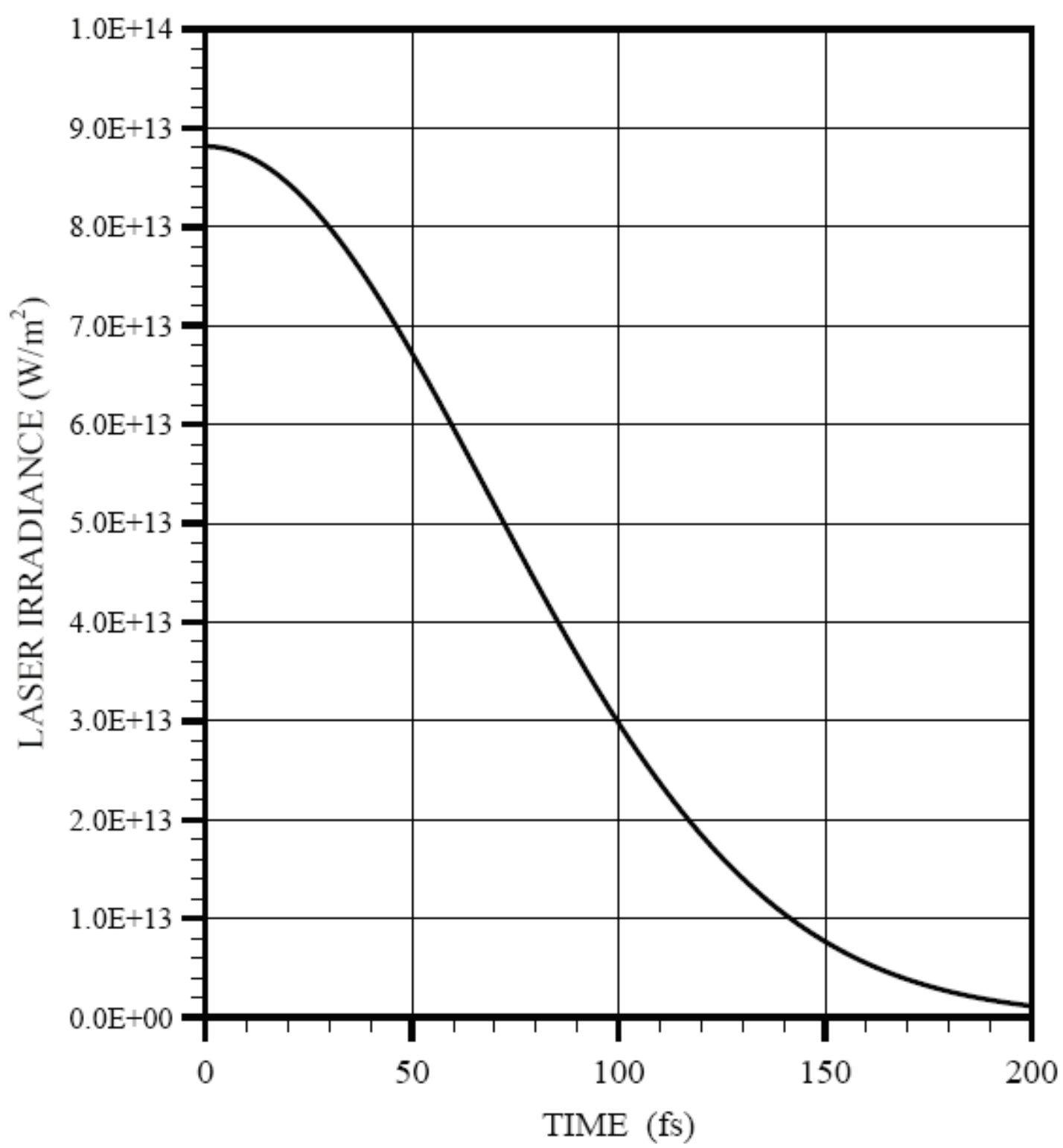

Fig. 2 Temporal variation of laser pulse intensity.

$\frac{d I}{I}=-\alpha(x) d x$

or integrating both sides yields,

$\Rightarrow \int_{I_{o}}^{I} \frac{d I}{I}=-\int_{0}^{L} \alpha(x) d x$

Due to the integration purpose, $x$ variable may be changed to another variable $u$. Also, to find the laser intensity at any point $x$ we may replace $L$ by $x$ in the above integration. Therefore,

$$
\Rightarrow \int_{I_{o}}^{I} \frac{d I}{I}=-\int_{0}^{x} \alpha(u) d u
$$

or

$$
\Rightarrow I=I_{o} e^{-\int_{0}^{x} \alpha(u) d u}
$$

Hence, the laser intensity at any point $x$ in the substrate material is given by,

$$
I=I_{o} e^{-\int_{0}^{x} \alpha(u) d u}
$$




\subsection{Volumetric Heat Generation}

When the thin film absorbs the laser beam energy, it generates heat. The heat generation rate is defined as,

$$
S=-\frac{d I}{d x}
$$

Substituting eq. 5 in eq. 6 and using Leibniz rule yields,

$$
S=-\frac{d}{d x}\left\{I_{o} e^{-\int_{0}^{x} \alpha(u) d u}\right\}
$$

Or

$$
S=-I_{o} e^{-\int_{0}^{x} \alpha(u) d u} \frac{d}{d x}\left\{-\int_{0}^{x} \alpha(u) d u\right\}
$$

Or

$$
S=I_{o} e^{-\int_{0}^{x} \alpha(u) d u}\left\{\int_{0}^{x} 0 \cdot d u+\alpha(x) \cdot 1-\alpha(0) \cdot 0\right\}
$$

Or

$$
S=I_{o} \alpha(x) e^{-\int_{0}^{x} \alpha(u) d u}
$$

Hence, the volumetric heat generation becomes:

$$
S=I_{o} \alpha e^{-\int_{0}^{x} \alpha(u) d u}
$$

The variable absorption coefficient can be determined after considering that absorption coefficient in the mathematical form of:

$$
\alpha_{1}=\alpha_{\max } \exp \left[-\left(\frac{x-b}{d}\right)^{2}\right]
$$

where b, and d are constants. Eq. 5 yields,

$$
I=I_{o} e^{-\int_{0}^{x} \alpha_{\max } \exp \left[-\left(\frac{u-b}{d}\right)^{2}\right] d u}
$$

Let $g(x)=\int_{0}^{x} \alpha_{\max } \exp \left[-\left(\frac{u-b}{d}\right)^{2}\right] d u$ then,

$$
g(x)=\frac{\sqrt{\pi}}{2} \alpha_{\max } d\left[\operatorname{erf}\left(\frac{x-b}{d}\right)-\operatorname{erf}\left(\frac{b}{d}\right)\right]
$$

The laser intensity and the volumetric heat generation are then given by,

$$
I=I_{o} e^{-g(x)}
$$

and

$$
S=I_{o} \alpha e^{-g(x)}
$$

Three cases are considered for the spatial distribution of absorption coefficient in the silicon film as shown in figure (3). It should be noted that the spatial distribution of absorption coefficient is based on the assumption that the presence of nano particles (to increase the absorption of laser intensity) in the silicon film. The cases are given below:

\subsection{Case 1: High Absorption in the surface region of} silicon

$\alpha_{1}=\alpha_{\max } \exp \left[-\left(\frac{x-b}{d}\right)^{2}\right] \& \boldsymbol{\alpha}(\boldsymbol{0})=\boldsymbol{\alpha}_{\max }$ and

$\alpha\left(l_{1}\right)=\alpha_{S i}$

Although laser pulse intensity distribution is not Gaussian, the absorption is assumed to be Gaussian for the simplicity. The constants $\mathrm{b}$ and $\mathrm{d}$ can be obtained from

$\alpha_{1}=\alpha_{\max } \exp \left[-\left(\frac{x-b}{d}\right)^{2}\right]$

after setting $\alpha(0)=\alpha_{\max }$ and $\alpha\left(l_{1}\right)=\alpha_{S i}$, i.e. the coefficients $b$ and $d$ are:

$$
b=0, \quad d=\frac{l_{1}}{\sqrt{\ln \left|\alpha_{\max } / \alpha_{S i}\right|}}
$$

2.5 Case 2: High Absorption in the mid-section of silicon

$$
\begin{aligned}
& \alpha_{2}=\alpha_{\max } \exp \left[-\left(\frac{x-b}{d}\right)^{2}\right] \& \alpha(l / 2)=\alpha_{\max }, \\
& \alpha(0)=\alpha\left(l_{1}\right)=\boldsymbol{\alpha}_{S i}
\end{aligned}
$$

The coefficients $b \& d$ are obtained from

$\alpha_{2}=\alpha_{\max } \exp \left[-\left(\frac{x-b}{d}\right)^{2}\right]$ 


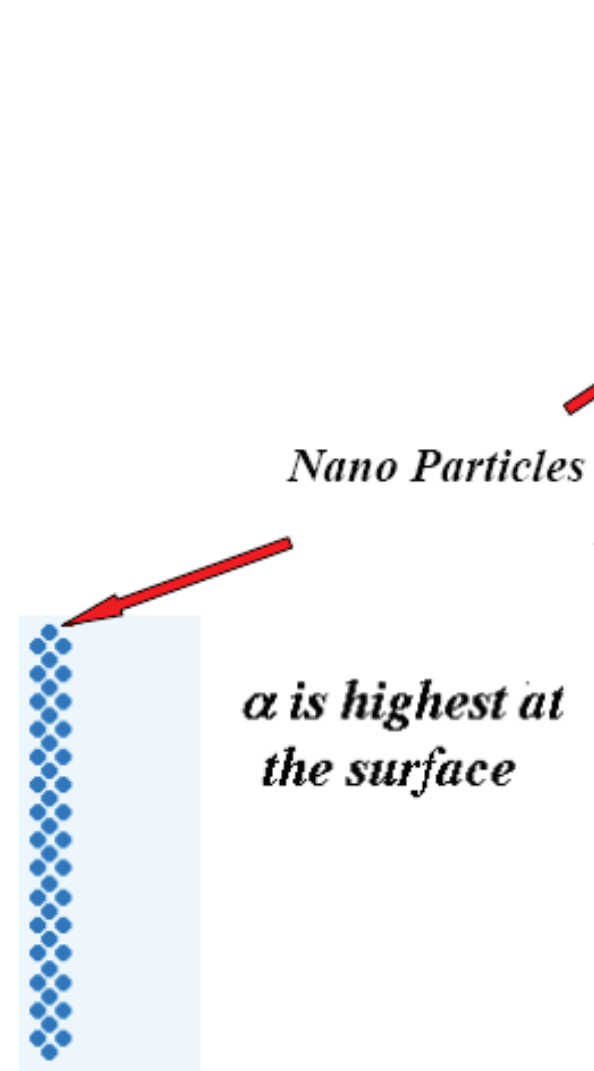

Case 1 $\alpha$ is highest

in the middle

\section{$\alpha$ is highest at the interface}

Fig. 3 Schematic view of the distribution of nano particles in silicon film.

after setting $\alpha\left(l_{1} / 2\right)=\alpha_{\max }, \alpha(0)=\alpha(L)=\alpha_{S i}$, i.e.:

$b=0, \quad d=\frac{0.5 l_{1}}{\sqrt{\ln \left|\alpha_{\max } / \alpha_{S i}\right|}}$

2.6 Case 3: High Absorption close to the gold-silicon interface

$\alpha_{3}=\alpha_{\max } \exp \left[-\left(\frac{x-b}{d}\right)^{2}\right] \& \alpha(0)=\alpha_{S i}$,

$\alpha\left(l_{1}\right)=\alpha_{\max }$

The coefficients $b \& d$ are obtained from

$\alpha_{3}=\alpha_{\max } \exp \left[-\left(\frac{x-b}{d}\right)^{2}\right]$

and $\alpha(0)=\alpha_{S i}, \alpha(L)=\alpha_{\max }$, the coefficients $b \& d$ are obtained as,

$$
b=a, \quad d=\frac{l_{1}}{\sqrt{\ln \left|\alpha_{\max } / \alpha_{S i}\right|}}
$$

\section{Initial and Boundary Conditions}

To solve eq. 1 , the following initial and boundary conditions are used. Initially (before the initiation of the laser pulse) the lattice and electron sub-systems are considered to be at the same initial temperature $\left(\mathrm{T}_{\mathrm{o}}\right)$, i.e.

At time $t=0: T_{l}=T_{o}$ and $T_{e}=T_{o}$

and

At time $\mathrm{t}=0: \frac{\partial T_{e}}{\partial t}=\frac{\partial T_{l}}{\partial t}=0$

The substrate material surface is considered to be insulated (no convection, nor radiation heat losses from the surface), i.e.:

At the surface at $\mathrm{x}=0: \frac{\partial T_{l}}{\partial x}=0$ and $\frac{\partial T_{e}}{\partial x}=0$

The substrate material is considered to be consisted of silicon layer on the top of the gold substrate with the silicon layer thickness of $l_{l}\left(l_{l}=1 \mu \mathrm{m}\right.$, figure (1)). At far distance away from the surface and inside gold, electron and lattice temperatures are assumed to reach equilibrium at temperature $\mathrm{T}_{0}$, i.e. at a large distance from the free surface $\mathrm{x}=1000 \mathrm{x} \delta(\delta$ being absorption depth $) \rightarrow \mathrm{T}_{1}=\mathrm{T}_{\mathrm{o}}$ and $\mathrm{T}_{\mathrm{e}}=$ $\mathrm{T}_{\mathrm{o}}$. However, at the interface of each layer, the continuity of flux and temperature are assumed, i.e.:

At the interface of the coating $\left(\mathrm{x}=l_{1}\right)$ :

$$
|T e|_{1}=|T e|_{2} \text { and }\left|T_{l}\right|_{1}=\left|T_{l}\right|_{2}
$$




$$
\left(\frac{\partial T_{e}}{\partial x}\right)_{1}=\frac{k_{2}}{k_{1}}\left(\frac{\partial T_{e}}{\partial x}\right)_{2} \text { and }\left(\frac{\partial T_{l}}{\partial x}\right)_{1}=\frac{k_{2}}{k_{1}}\left(\frac{\partial T_{l}}{\partial x}\right)_{2}
$$

where the indices 1 and 2 represent silicon and gold, respectively.

\section{Numerical Solution}

The numerical method employed to solve the energy transport equation (eq. 1) uses a finite difference scheme, which is well established in the literature [16]. In order to obtain accurate results, the convergence criteria should be met [17]. The thermophysical properties of silicon and gold are given in Table 1 .

Table 1 Properties used in the simulations

\begin{tabular}{lcc}
\hline Parameter & Silicon & Gold \\
\hline Material Thickness $(\mu \mathrm{m})$ & 1 & 0.45 \\
$\begin{array}{l}\text { Electron Heat Capacity, } \mathrm{C}_{\mathrm{e}} \\
\left(\mathrm{J} / \mathrm{m}^{3} / \mathrm{K}\right)\end{array}$ & $2.1 \times 10^{4}$ & $2.1 \times 10^{4}$ \\
$\begin{array}{l}\text { Lattice Heat Capacity, } \mathrm{C}_{\mathrm{l}} \\
\left(\mathrm{J} / \mathrm{m}^{3} / \mathrm{K}\right)\end{array}$ & $1.65 \times 10^{6}$ & $2.5 \times 10^{6}$ \\
$\begin{array}{l}\text { Thermal Conductivity, } \mathrm{k} \\
(\mathrm{W} / \mathrm{m} / \mathrm{K})\end{array}$ & 130 & 315 \\
$\begin{array}{l}\text { Coupling Constant, G } \\
\left(\mathrm{W} / \mathrm{m}^{3} / \mathrm{K}\right)\end{array}$ & $136.6 \times 10^{16}$ & $2.6 \times 10^{16}$ \\
Absorption Coefficient, $\delta\left(\mathrm{m}^{-1}\right)$ & 1000 & $6.17 \times 10^{7}$ \\
\hline
\end{tabular}

\section{Results and Discussion}

Laser short-pulse heating of silicon film located at the surface of aluminium substrate is considered. The absorption coefficient in the film is varied spatially resembling the addition of metallic nano-particles in the silicon film. The study is extended to include three different distributions of absorption coefficient in the silicon film.

Figure (4) shows spatial distribution of absorption coefficient and the corresponding laser irradiance and volumetric heat generation in the silicon film. It should be noted that the spatial distributions of the absorption coefficient (eq.8) is selected to obtain, i) high absorption in the surface vicinity of the silicon film, ii) high absorption in the mid-section of the silicon film, and iii) high absorption in the silicon film close to the aluminium substrate. The spatial distribution laser irradiance intensity is obtained using the Lambert's Beer Law (eq.11) while the volumetric heat generation rate is computed from eq.12. It is evident that absorption in the surface vicinity of the film results in rapid decay of laser intensity with increasing depth below the film surface. However, this decay is gradual for the case of high absorption in the mid-section and in the region close to the aluminium interface. This is because of the transmittivity of silicon film for the wave length of incident irradiation, which is high. Moreover, high absorption coefficient in the surface region results in high rate of volumetric heat generation in the film. Consequently, volumetric heat generation becomes the maximum in the vicinity of the free surface of the silicon film for the absorption coefficient distribution, which has the maximum value at the surface.
Figure (5) shows electron temperature distribution inside the silicon film for three different absorption coefficient distributions and three heating periods. Electron temperature attains high values in the region where the absorption is high, which is more pronounced for $40 \mathrm{fs}$ of the heating duration. In this case, progressing time during the heating pulse (as shown in figure (2)) results in increased electron excess energy due to absorption from the irradiated field. Although electrons loss the fraction of their excess energy to the lattice site through successive collisions during the heating period, electron excess energy loss is not significant due to the short heating duration. However, temperature decays sharply in the surface region for the distribution of absorption coefficient resulting in high absorption in the free surface region of the film. This is because of the energy gain of electrons in the surface vicinity from the irradiated field through absorption. Moreover, the decay of laser intensity is relatively sharper in the surface region as compared to those distributions of absorption coefficient. Consequently, the sharp decay of laser intensity in the surface region is responsible for the sharp decay of electron excess energy in the surface region of the film. Although collisional energy transport from electron sub-system to lattice sub-system reduces electron temperature, this reduction is not significant due to a few numbers of collisions during the short heating period. Electron temperature distribution inside the film changes as the distribution of absorption coefficient in the film changes. In this case, electron temperature increases gradually to reach its maximum where the volumetric heat generation is the maximum. It is also evident from the intensity distribution curve (figure (4)) that intensity decays gradually across the high absorption region in the film. Consequently, energy absorbed by the electrons from the irradiated field decays gradually with increasing depth in this region. This, in turn, lowers electron temperature with increasing depth in this region. However, gradual increase of electron temperature before reaching its maximum is associated with electron excess energy gain in this region, since absorption coefficient increases gradually before reaching it speak value, i.e. gradual increase of absorption coefficient results in gradual increase of electron excess energy and electron temperature. When comparing the maximum electron temperatures due to the different distributions of absorption coefficient in the film, it is evident that the highest electron temperature occurs when the laser incident energy is absorbed in the surface region of the film. However, as the high absorption region moves in to the film, electron temperature reduces considerably.

Figure (6) shows temporal variation of electron temperature for three different distributions of the absorption coefficient. It should be noted that three locations inside the silicon film are selected to demonstrate electron temperature distributions. The selection of the locations is based on the maximum electron temperature inside the film (figure (5)). Electron temperature increases sharply with progressing time for the case that the absorption coefficient is the maximum at the surface. However, the gradual increase in electron temperature occurs as the location of the high absorption region moves inside the film. Moreover, the rapid increase in electron temperature at the surface associated with the energy gain 
JLMN-Journal of Laser Micro/Nanoengineering Vol. 7, No. 2, 2012
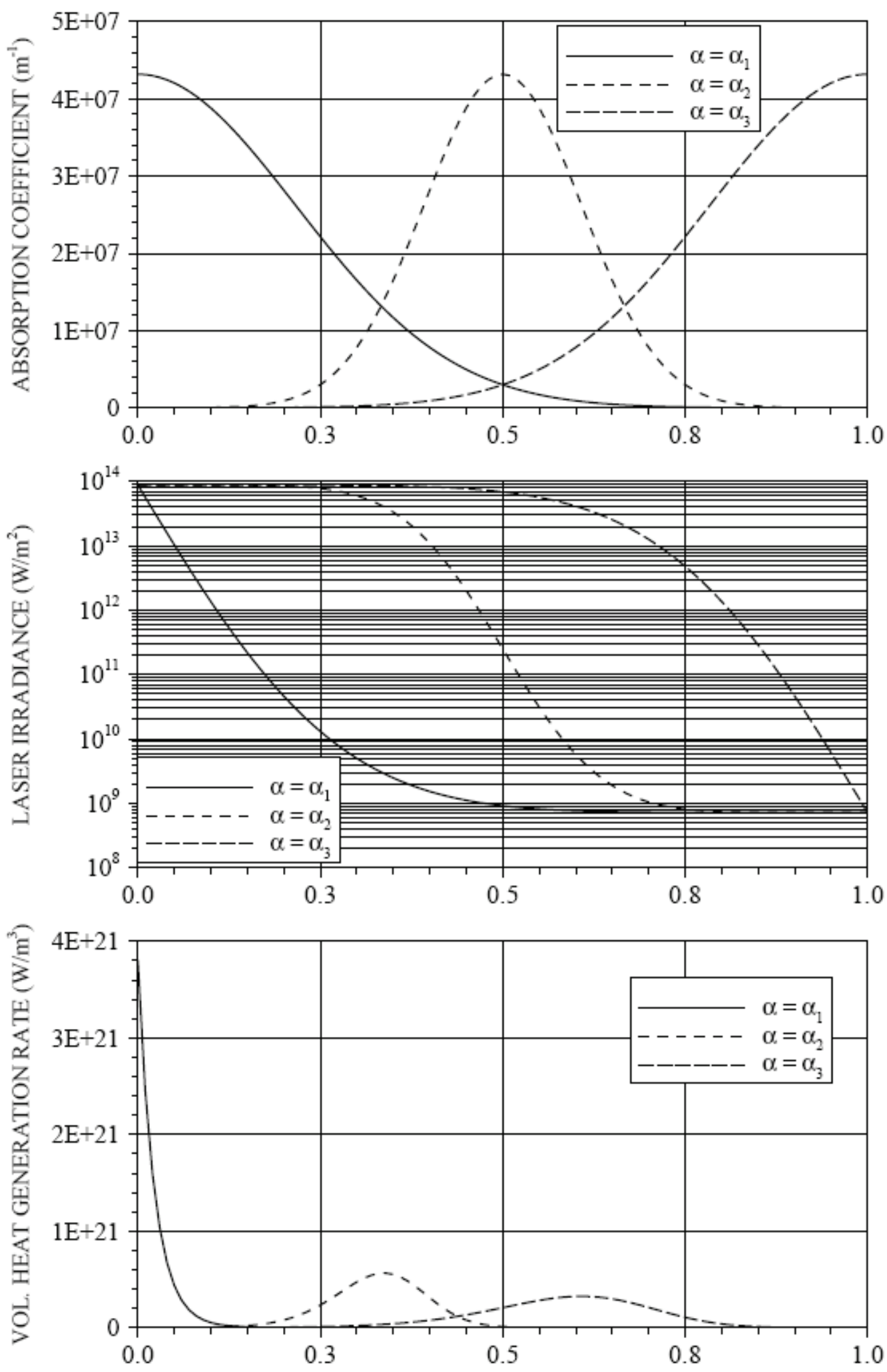

DISTANCE ALONG THE FILM THICKNESS $(\mu \mathrm{m})$

Fig. 4 Distributions of absorption coefficient and corresponding laser intensity and volumetric heat generation in the silicon film for three cases. 

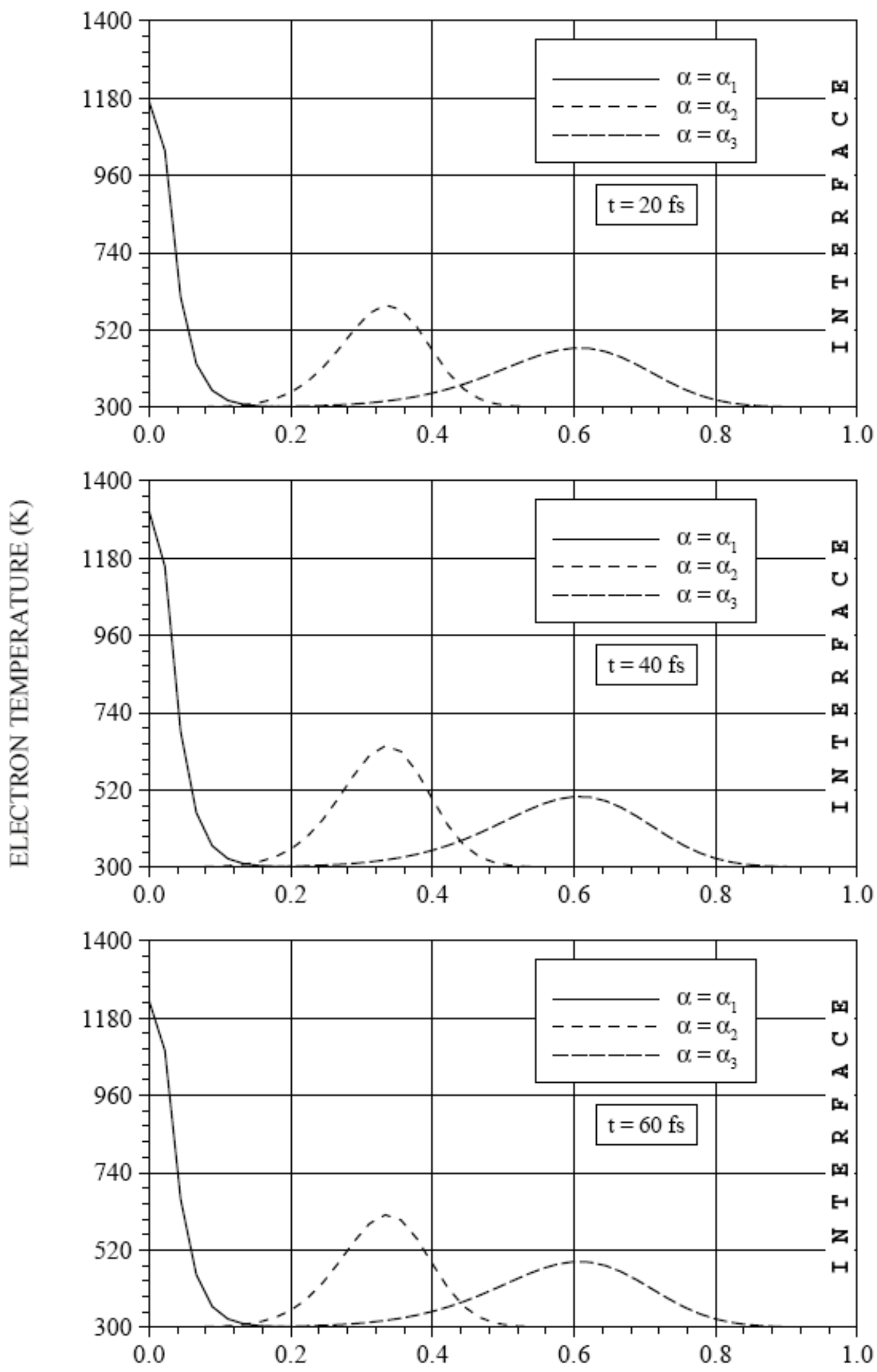

DISTANCE ALONG THE FILM THICKNESS $(\mu \mathrm{m})$

Fig. 5 Electron temperature distributions in the silicon film for three cases of absorption coefficient distributions. 


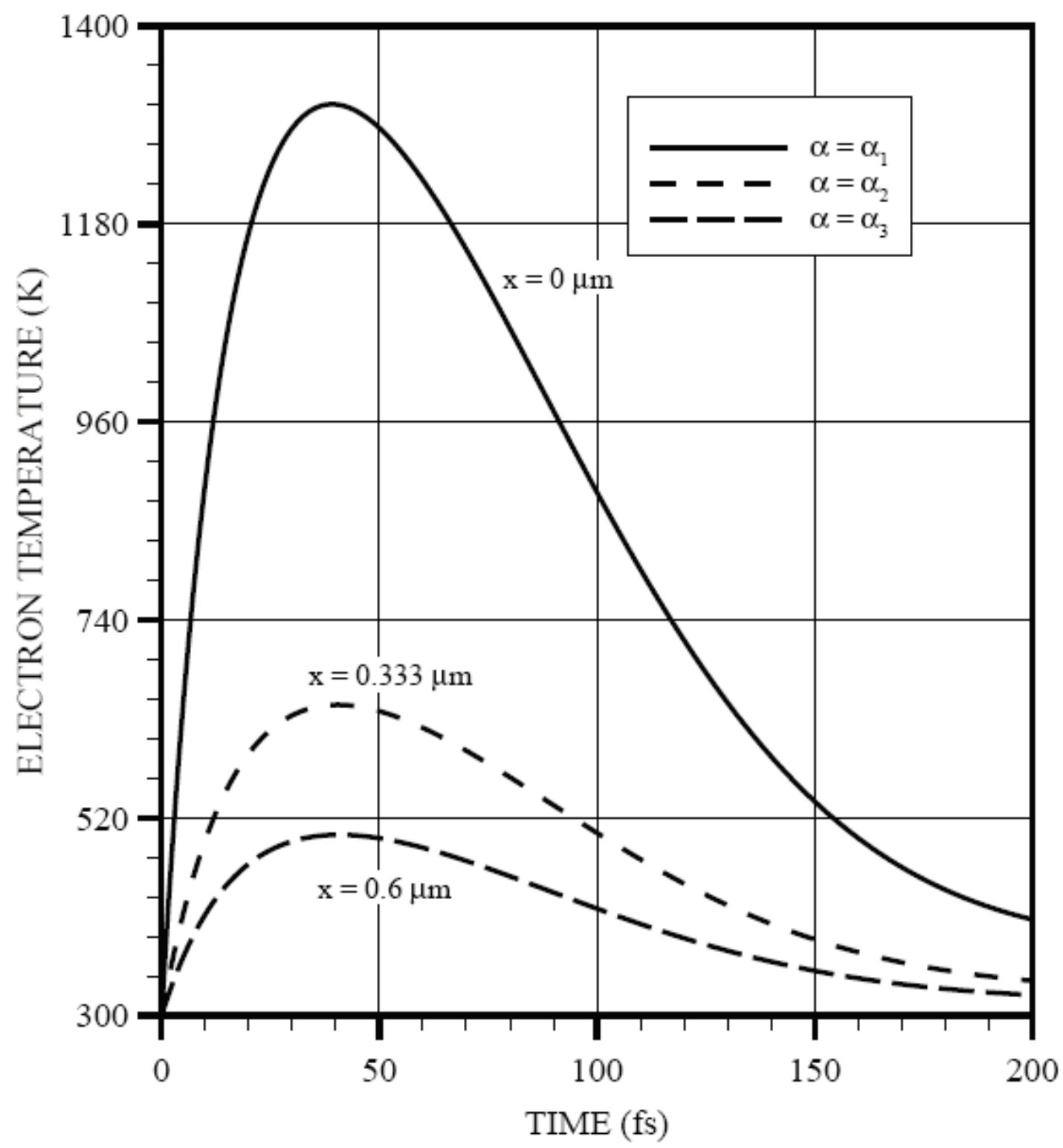

Fig. 6 Temporal variation of electron temperature at three locations in the silicon film and for three cases of absorption coefficient distributions.

of electrons from the irradiated filed, which has high energy content due to high absorption coefficient at the surface. As the location of high temperature region moves in to the film, gradual increase in the absorption coefficient results in less irradiated energy reaching the peak absorption region. Consequently, low irradiated intensity (figure (4)), results in gradual increase in electron temperature. As the time progresses, the rate of electron temperature increase becomes less as compared to that corresponding to the initial heating period ( $t \leq 20 \mathrm{fs}$ ). This is attributed to one or all of the followings: i) electron excess energy dissipation through electron diffusion increases as electron temperature increases, ii) the amount of electron excess energy transfer to lattice site increases as electron excess energy increases and the number of collisions between electrons and lattice site increases with progressing time ( $\mathrm{t}>20 \mathrm{fs})$. Moreover, the decay rate of electron temperature from its maximum changes with the location of the high absorption region in the film. In this case, electron temperature decays sharply for the case of the high absorption in the surface region of the film.

Figure (7) shows lattice site temperature distribution inside the film for three different absorption coefficient distributions and three heating periods. Lattice site temperature distributions are similar to electron temperature distributions for all the cases considered. This is because of energy gain of the lattice sub-system from electrons. Lattice site energy increases as electron excess energy transfers through the collisional process. Consequently, electrons with high excess energy results in high lattice temperature. Temperature decays sharp and resulting the high temperature gradient for the case of high absorption in the surface region. This, in turn, slightly enhances the diffusional energy transfer from the surface region to its neighbourhood in the silicon film, despite the 

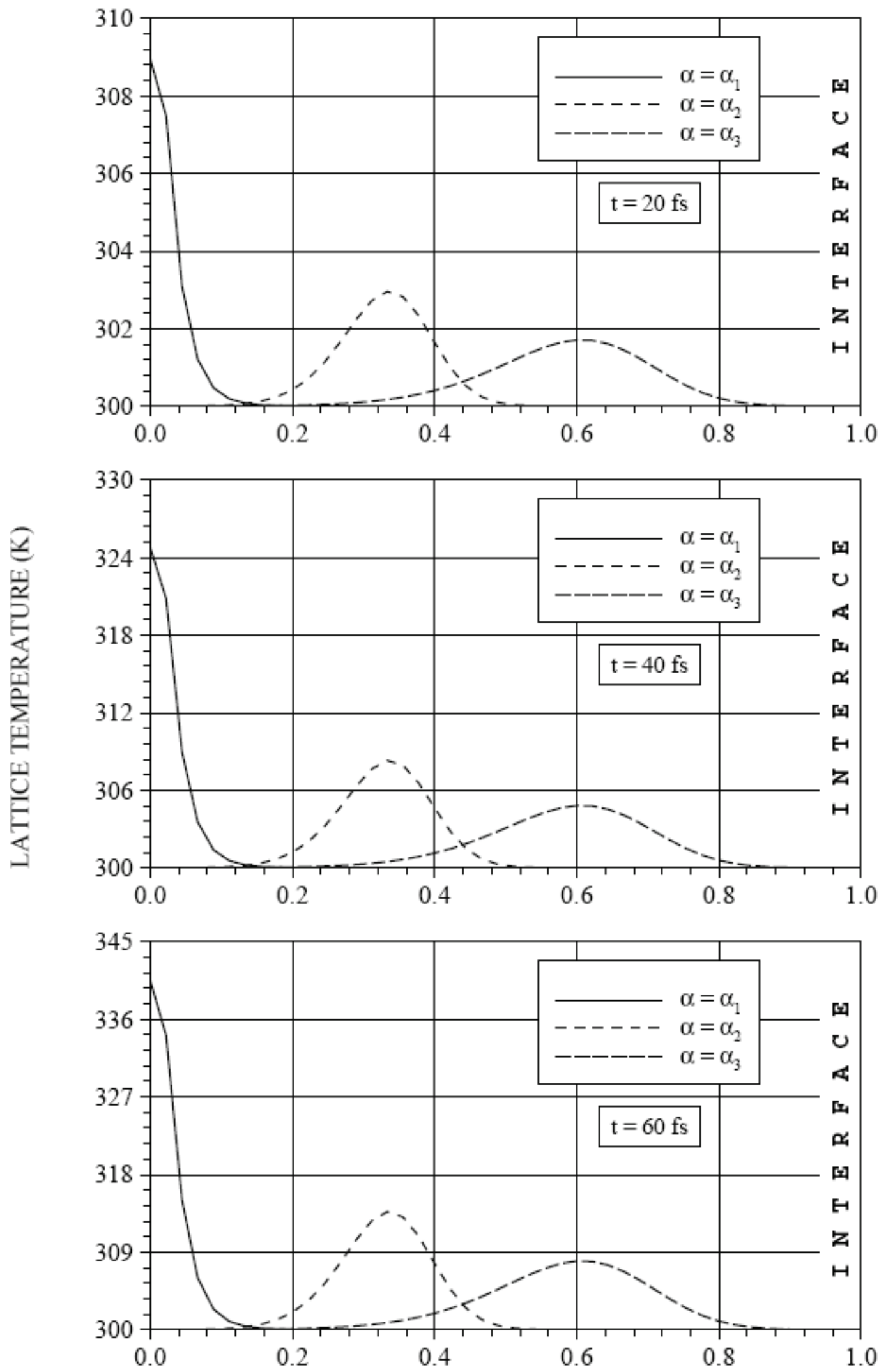

DISTANCE ALONG THE FILM THICKNESS $(\mu \mathrm{m})$

Fig. 7 Lattice site temperature distributions in the silicon film for three cases of absorption coefficient distributions. 


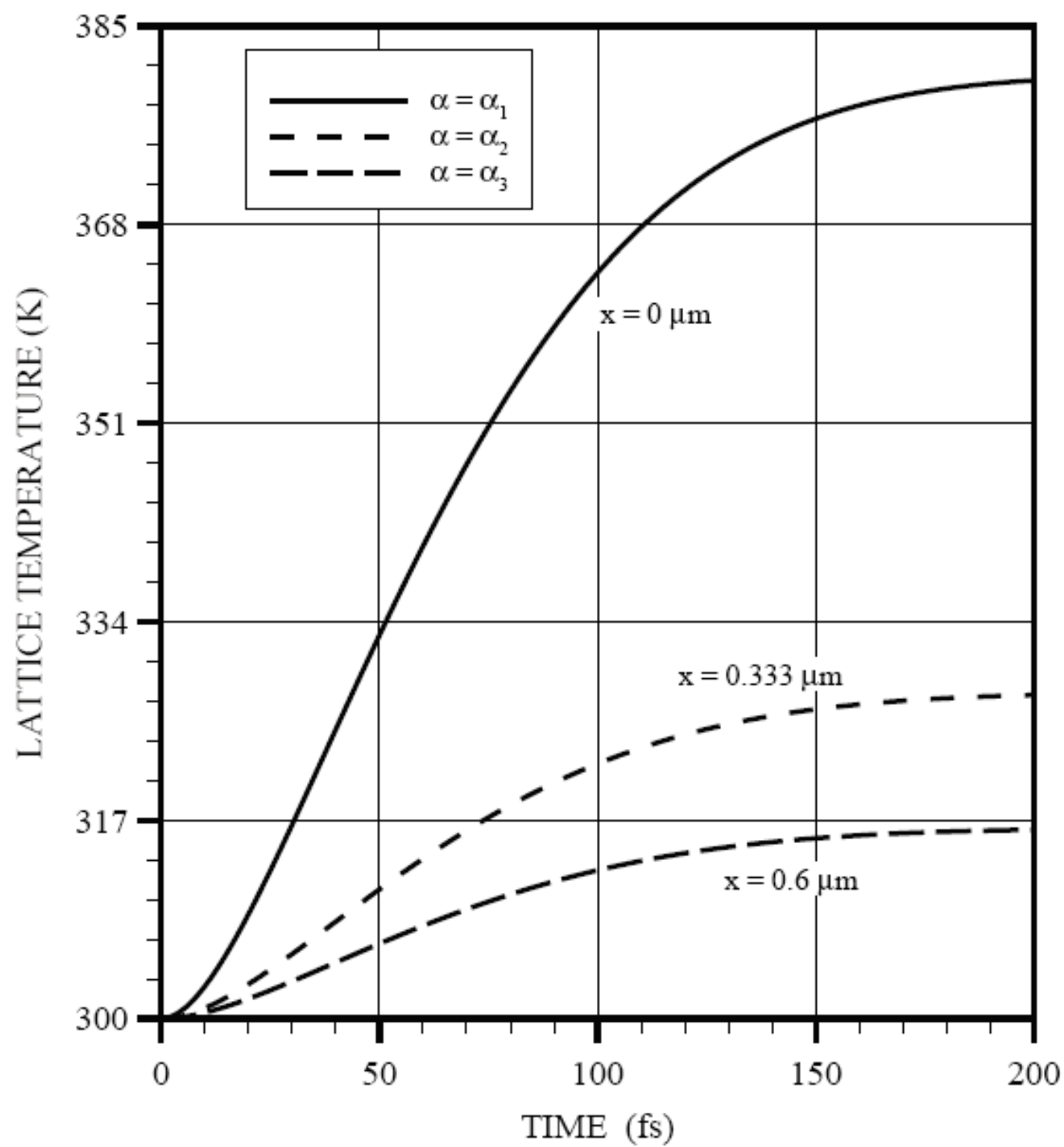

Fig. 8 Temporal variation of lattice site temperature at three locations in the silicon film and for three cases of absorption coefficient distributions.

fact that the heating duration is short and this process does not have significant effect on lattice temperature in the surface vicinity. Unlike the peak electron temperature, which reduces at $60 \mathrm{fs}$ heating time, lattice temperature increases with progressing time. This is because of lattice site energy gain from the electron sub-system through the collisional process. It should be noted that lattice site temperature increase continuous until electron and lattice sub-systems comes into a thermal equilibrium. The increase in lattice site temperature is less for the case of high absorption coefficient located inside the film with progressing time. This is because of less electron excess energy and temperature in this region (figure (5)).

Figure (8) shows temporal variation of lattice site temperature at different locations where the absorption is high in the film and for different distribution of the absorption coefficients. The rise of lattice temperature is fast at the surface. As the location of high absorption region moves inside of the silicon film, the rate of rise of lattice temperature reduces. Moreover, lattice site temperature rise is small in the early heating period $(\mathrm{t} \leq 20 \mathrm{fs}$ at $\mathrm{x}=0 \mu \mathrm{m}$ location) despite the fact that electron excess energy and temperature increase is rapid (figure (6)). This is because of electron excess energy transfer to lattice site through the collisional process; in which case, the number of collisions in the early heating period is small, i.e. only few collisions. However, as the heating time progresses, the number of collisions increases so that electron excess energy transfer to lattice site increases. This lowers the rate of electron temperature during this period (figure (6)). Moreover, lattice site temperature increase becomes gradual as the heating period progresses further $(\mathrm{t}>100$ fs at $\mathrm{x}=0$ 


\section{JLMN-Journal of Laser Micro/Nanoengineering Vol. 7, No. 2, 2012}

location). This is attributed to electron excess energy drop during this period (figure (6)); hence, the amount of electron excess energy transfer to lattice site becomes less, despite that the number of collisions increases. It should be noted that as the laser pulse ends, electron energy gain from the irradiated field ceases. This drops electron excess energy considerably.

\section{Conclusion}

Laser short-pulse heating of silicon film located next to the aluminium plate is examined. The spatial distribution of absorption coefficient is varied in the silicon film to investigate its influence on electron and lattice site temperature distribution. The spatial distribution of absorption coefficient in the film is selected to resemble the distribution of absorbed particles at different locations in the film. It is found hat electron temperature attains the highest value for the high absorption region located in the surface vicinity. As the high absorption region moves into the film, electron temperature reduces considerably, which is attributed to the laser intensity distribution in this region. Electron temperature rises sharply in the early heating period, which is more pronounced for the high absorption region located in the surface region of the film. As the heating period progresses further, laser pulse ceases. This, in turn, results in decay of electron excess energy in the irradiated region. However, the rate of decay is high for the high absorption region located in the surface region of the film. The decay of electron energy and temperature after the pulse ending is associated with the collisional energy transport to lattice site in this region. Lattice site temperature rises at low rate in the early heating period, despite the high electron excess energy. In this case, small number of collisions suppresses energy transfer to lattice site while lowering lattice site temperature rise during this period. As the time progresses, lattice site temperature increases at a high rate due to increase in the number of electron-lattice collisions. As the time increases further (beyond the laser pulse ending), lattice site temperature increase becomes gradual due to drop in electron excess energy during this period. Lattice temperature attains high values in the film where electron temperature is high. As the high absorption region moves into the film, the maximum lattice site temperature reduces considerably.

\section{Acknowledgements}

The authors acknowledge the support of King Fahd University of Petroleum and Minerals. Dhahran, Saudi Arabia for this work.

\section{References}

[1] G.L. Eesley, Observation of Nonequilibtium Electron Heating in Copper, Phys. Rev. Lett. 51 (1983) 2140-2143.

[2] S.D. Brorson, J.G. Fujimoto, and E.P. Ippen, Femtosecond Electronic Heat-Transport Dynamic in Thin Gold Films, Phys. Rev. Lett. 59 (1987)1962-1965.

[3] X.Y. Wang, D.M. Riffe, Y.S. Lee, and M.C. Downer, Time-resolved electron-temperature measurement in a highly excited gold target using femtosecond thermionic emission, Phys. Rev. B, 50 (1994) 8016-8019.
[4] G. Chen, C. L. Tien, Partial Coherence Theory of Thin Film Radiative Properties, Journal of Heat Transfer 114 (1992) 636-643.

[5] L. Malinowski, A relaxation model for heat conduction and generation, J. Phys. D: Appl. Phys. 26 (1993) 11761180 .

[6] T. Q. Qiu, T. Juhasz, C. Suarez, W. E. Bron, C. L. Tien, Femtosecond laser heating of multi-layer metals - II. Experiments, International Journal of Heat \& Mass Transfer 37 (17) (1994) 2799-2808.

[7] W. Rozmus, V. T. Tikhonchuk, A model of ultrashort laser pulse absorption in solid targets, Phys. Plasmas 3 (1) (1996) 360-366.

[8] M. A. Al-Nimr, S. A. Masoud, Nonequilibrium Laser Heating of Metal Films, Journal of Heat Transfer 119 (1997) 188-190.

[9] M. Honner, J. Kunes, On the Wave Diffusion and Parallel Nonequilibrium Heat Conduction, Journal of Heat Transfer 121 (1999) 702-707.

[10] L. Jiang, Hai-Lung Tsai, Improved Two-Temperature Model and Its Application in Ultrashort Laser Heating of Metal Films, Journal of Heat Transfer 127 (2005) 11671173.

[11] L. Pilon, K. M. Katika, Modified Method of Characteristics for Simulating Microscale Energy Transport, Journal of Heat Transfer 126 (2004) 735-743.

[12] W. An, L. M. Ruan, H. P. Tan, H. Qi, Y. M. Lew, Finite Element Simulation for Short Pulse Light Radiative Transfer $n$ Homogenous and Nonhomogenous Media, Journal of Heat Transfer 129 (2007) 353-362.

[13] B.S. Yilbas, Laser Short-Pulse Heating: ThreeDimensional Consideration, Proc Instn Mech Engrs Part C 215 (2001) 1123-1138.

[14] B.S. Yilbas and S.Z. Shuja, Laser heating of silicon: a kinetic theory approach, Numerical Heat Transfer Part A. 36(1999) 563-584.

[15] B.S. Yilbas, Laser shortpulse heating of gold: variable properties case, Int. J. Heat and Mass Transfer 46(2003) 3511-3520.

[16] B.S. Yilbas, Improved formulation of electron kinetic theory approach for laser short-pulse heating", Int. J. Heat and Mass Transfer 49 (2006) 2227-2238.

[17] G.D. Smith, Numerical solution of partial differential equations: finite difference methods, 3rd edn, Clarendon Press, Oxford, 1985 
JLMN-Journal of Laser Micro/Nanoengineering Vol. 7, No. 2, 2012

\section{Nomenclature}

b: constant in absorption equation (eq.8)

$\mathrm{C}_{\mathrm{e}}$ : Electron heat capacity $\left(\mathrm{J} / \mathrm{m}^{3} \mathrm{~K}\right)$

$\mathrm{C}_{1}$ : Lattice heat capacity $\left(\mathrm{J} / \mathrm{m}^{3} \mathrm{~K}\right)$

$\mathrm{Cp}$ : Specific heat of lattice site (J/kg.K)

$\mathrm{d}$ : constant in absorption equation (eq.8)

f: Fraction electron excess energy transfer to lattice site during a single collision

$\mathrm{I}$ : Laser intensity $\left(\mathrm{W} / \mathrm{m}^{2}\right)$

$\mathrm{I}_{\mathrm{o}}$ : Laser peak power intensity $\left(\mathrm{W} / \mathrm{m}^{2}\right)$

$\mathrm{J}$ : Energy Flux $\left(\mathrm{J} / \mathrm{m}^{2}\right)$

$\mathrm{k}$ : Thermal conductivity $(\mathrm{W} / \mathrm{m} . \mathrm{K})$

S: Volumetric heat generation $\left(\mathrm{W} / \mathrm{m}^{3}\right)$

$l_{l}$ : Silicon film thickness $(\mathrm{m})$

$\mathrm{T}_{1}$ : Lattice site temperature $(\mathrm{K})$
$\mathrm{T}_{\mathrm{e}}$ : Electron temperature $(\mathrm{K})$

t: Time (s)

$t_{\mathrm{p}}$ : Laser pulse length (s)

$\mathrm{x}$ : Spatial coordinates corresponding to the $\mathrm{x}$-axis for phonon (m)

\section{Greek letters}

$\alpha:$ Absorption coefficient $(1 / \mathrm{m})$

$\lambda$ : Mean free path of electrons $(\mathrm{m})$

$\rho:$ Density $\left(\mathrm{kg} / \mathrm{m}^{3}\right)$

$\tau_{\mathrm{p}}$ : Electron mean free time between electron-phonon coupling (s)

$\tau_{\mathrm{s}}:$ Electron-phonon characteristic time $\left(\tau_{\mathrm{s}}=\mathrm{Ce} / \mathrm{G}\right)(\mathrm{s})$

(Received: January 17, 2011, Accepted: April 05, 2012) 